Received: 14.08 .2021

Revised: 14.10 .2021

Accepted: 29.10 .2021

DOI: $10.17804 / 2410-9908.2021 .6 .60-71$

\title{
PARALLEL TECHNOLOGY FOR SOLVING NONSTATIONARY HEAT CONDUCTION PROBLEMS IN AXISYMMETRIC DOMAINS
}

\author{
L. F. Spevak ${ }^{\text {a) }}$, O. A. Nefedova ${ }^{\text {b)* }}$ \\ Institute of Engineering Science, Ural Branch of the Russian Academy of Sciences, \\ 34 Komsomolskaya St., Ekaterinburg, 620049, Russian Federation \\ a) iD http://orcid.org/0000-0003-2957-6962 《1fs@imach.uran.ru; \\ b) (iD http://orcid.org/0000-0003-1163-6699 nefedova@imach.uran.ru \\ *Corresponding author. E-mail: nefedova@imach.uran.ru \\ Address for correspondence: 34 Komsomolskaya St., Ekaterinburg, 620049, Russian Federation \\ Tel.: +7 (343) 36230 22; fax: +7 (343) 3745330
}

The paper develops a parallel algorithm and program for solving nonstationary heat conduction and diffusion problems in axisymmetric domains with axisymmetric boundary conditions. The numerical solution is based on the boundary element method. In order to optimize and enhance the effectiveness of the computer implementation of the algorithm, the computations are parallelized and the OpenMP application program interface is used. The program is tested by comparing the calculation results with the data of known exact solutions. The calculations confirm the correctness of the numerical solutions and the possibility of full scaling at different numbers of boundary elements according to the number of cores/processors available. The program is applicable to solving axisymmetric heat conduction and diffusion problems and, as a component of a software system, to solving nonlinear problems.

Keywords: axisymmetric heat conduction problem, boundary element method, parallel computations, OpenMP.

\section{Acknowledgment}

The work was performed under a state assignment, state registration number AAAA-A18118020790140-5.

\section{References}

1. Fedotov V.P., Spevak L.F., Nefedova O.A. Parallel algorithms for strength analysis of hydrogenated structures. Programmnye produkty $i$ sistemy, 2012, vol. 99, No. 3, pp. 235-239. (In Russian).

2. Fedotov V.P., Spevak L.F., Nefedova O.A. A software package designed to solve problems of the potential theory by the boundary element method Programmnye produkty i sistemy, 2014, vol. 108, No. 4, pp. 178-183. DOI: 10.15827/0236-235X.108.178-182. (In Russian).

3. Fedotov V.P., Spevak L.F. Analytical integration of kernel functions for solving elasticity problems and potential theory by the method of boundary elements. Matematicheskoe modelirovanie, 2007, vol. 19, No. 2, pp. 87-104. (In Russian).

4. Fedotov V.P., Spevak L.F. One approach to the derivation of exact integration formulae in the boundary element method. Engineering Analysis with Boundary Elements, 2008, vol. 32, No. 10, pp. 883-888. DOI: 10.1016/j.enganabound.2008.03.001. 
5. $\quad$ Fedotov V.P., Spevak L.F., Nefedova O.A. Моделирование процессов упругопластического деформирования модифицированным методом граничных элементов. Programmnye produkty i sistemy, 2013, vol. 4, No. 4, pp. 253-257. (In Russian).

6. Spevak L.F., Nefedova O.A. Solving a two-dimensional nonlinear heat conduction equation with degeneration by the boundary element method with the application of the dual reciprocity method. AIP Conference Proceedings, 2016, vol. 1785, 040077. DOI: 10.1063/1.4967134.

7. Spevak L.F., Nefedova O.A. Solving a two-dimensional nonlinear heat conduction equation with nonzero boundary conditions by the boundary element method. AIP Conference Proceedings, 2017, vol. 1915, 040055. DOI: 10.1063/1.5017403.

8. Kazakov A.L., Nefedova O.A., Spevak L.F. Solution of the Problem of Initiating the Heat Wave for a Nonlinear Heat Conduction Equation Using the Boundary Element Method. Computational Mathematics and Mathematical Physics, 2019, vol. 59, iss. 6, pp. 1015-1029. DOI: $10.1134 / \mathrm{S} 0965542519060083$.

9. Kazakov A., Spevak L., Nefedova O., Lempert A. On the Analytical and Numerical Study of a Two-Dimensional Nonlinear Heat Equation with a Source Term. Symmetry-Basel, 2020, vol. 12, iss. 6, article 921. DOI: 10.3390/sym12060921.

10. Kazakov A.L., Spevak L.F., Nefedova O.A. A Numerical Solution to the Two-Dimensional Nonlinear Degenerate Heat Conduction Equation with a Source. AIP Conference Proceedings, 2020, vol. 2315, 040018. DOI: 10.1063/5.0036718.

11. Spevak L.F., Nefedova O.A. Parallel technology for solving the poisson equation in axisymmetric domains by the boundary element method. AIP Conference Proceedings, 2018, vol. 2053, 030070. DOI: 10.1063/1.5084431.

12. Nefedova O.A., Spevak L.F. Parallel Technology for Solving Axisymmetric Problems of the Theory of Elasticity by the Boundary Element Method. AIP Conference Proceedings, 2020, vol. 2315, 020030. DOI: 10.1063/5.0037021.

13. Rizzo F.J., Shippy D.J. A method of solution for certain problems of transient heat conduction. AIAA J., 1970, vol. 8, pp. 2004-2009. DOI:10.2514/3.6038.

14. Shaw R.P. An integral equation approach to diffusion. International Journal of Heat and Mass Transfer, 1974, vol. 17 (6), pp. 693-699. DOI: 10.1016/0017-9310(74)90202-6.

15. Brebbia C.A., Walker S. Boundary Element Techniques in Engineering, NewnesButterworths, London, 1980. ISBN: 9781483102566.

16. Wrobel L.C., Brebbia C.A. A formulation of the boundary element method for axisymmetric transient heat conduction. International Journal of Heat and Mass Transfer, 1981, vol. 24, pp. 843850. DOI: 10.1016/S0017-9310(81)80007-5.

17. Zhu S.P. Time-dependent reaction diffusion problems and the LTDRM approach. In: M. Goldberg, ed. Boundary Integral Methods: Numerical and Mathematical Aspects. Computational Mechanics Publications, Southampton, Boston, 1999, pp. 1-35.

18. Sutradhar A, Paulino G.H, Gray L.J. Transient heat conduction in homogeneous and nonhomogeneous materials by the Laplace Transform Galerkin boundary element method. Engineering Analysis with Boundary Elements, vol. 26 (2), pp. 119-132. DOI: 10.1016/S09557997(01)00090-X.

19. Brebbia C.A., Telles J.F.C., Wrobel L.C. Boundary Element Techniques, Springer-Verlag, Berlin, Neidelberg, New-York, Tokyo, 1984, 466 p. ISBN: 978-3-642-48862-7 (print), 978-3-64248860-3 (online). DOI: 10.1007/978-3-642-48860-3.

20. Abramowitz M., Stegun I.A. Handbook of Mathematical Functions with Formulas, Graphs, and Mathematical Tables. In: M. Abramowitz, I. A. Stegun, eds. Dover Books on Advanced Mathematics, Dover Publications, New York, 1965.

21. Kronrod A.S. Uzly i vesa kvadraturnykh formul [Nodes and weights of quadrature formulas]. Moscow, Nauka Publ., 1964, 143 p. (In Russian). 
22. Lifanov I.K. Metod singulyarnykh integralnykh uravnenii $i$ chislennyi eksperiment [Singular integral equations method and numerical experiment]. Moscow, Yanus Publ., 1995, 520 p. ISBN 5-88929-003-7. (In Russian).

23. GSL-GNU Scientific Library. Available at: http://www.gnu.org/software/gsl/ (accessed 07.04.2021).

24. Boost C++ Libraries. Available at: http://www.boost.org/ (accessed 07.04.2021).

25. What is OpenMP? PARALLEL.RU. Available at: https://parallel.ru/tech/tech_dev/openmp.html (accessed 11.05.2021).

26. OpenMP. Available at: http://www.openmp.org/ (accessed 11.05.2021).

27. Lykov A.V. Teoriya teploprovodnosti [Heat conduction theory]. Moscow, Vysshaya shkola Publ., 1967, 597 p. (In Russian). 
Подана в журнал: 14.08.2021

УДК 519.633

DOI: $10.17804 / 2410-9908.2021 .6 .60-71$

\title{
ПАРАЛЛЕЛЬНАЯ ТЕХНОЛОГИЯ РЕШЕНИЯ НЕСТАЦИОНАРНЫХ ЗАДАЧ ТЕП- ЛОПРОВОДНОСТИ В ОСЕСИММЕТРИЧНОЙ ПОСТАНОВКЕ
}

\author{
Л. Ф. Спевак ${ }^{\text {a)}}$, О. А. Нефедова ${ }^{\text {б** }}$ \\ Институт машиноведения УрО РАН, \\ 34, ул. Комсомольская, г. Екатеринбург, Российская Федерация \\ a) iD http://orcid.org/0000-0003-2957-6962 1fs@imach.uran.ru; \\ б) iD http://orcid.org/0000-0003-1163-6699 nefedova@imach.uran.ru \\ *Ответственный автор. Эл. почта: nefedova@imach.uran.ru \\ Адрес для переписки: ул. Комсомольская, 34, Екатеринбург, Российская Федерация \\ Тел.: +7 (343) 362-30-22; факс: +7 (343) 374-53-30
}

Работа посвящена разработке параллельного алгоритма и программы для решения нестационарных задач теплопроводности и диффузии в осесимметричных областях при осесимметричных граничных условиях. В основе численного решения лежит метод граничных элементов. Для оптимизации и повышения эффективности компьютерной реализации алгоритма выполнено распараллеливание вычислений и привлечен открытый стандарт параллельного программирования ОреnMP. Разработанная программа была протестирована сравнением результатов расчетов с данными известных точных решений. Расчеты подтверждают корректность численных решений и возможность полного масштабирования при различных количествах граничных элементов в соответствии с количеством доступных ядер/процессоров. Программа может быть использована для решения осесимметричных задач теплопроводности и диффузии, а также как составляющая программного комплекса для решения нелинейных задач.

Ключевые слова: осесимметричная задача теплопроводности, метод граничных элементов, параллельные вычисления, OpenMP.

\section{1. Введение}

Задача разработки специализированных программных средств для решения краевых задач математической физики не теряет своей актуальности. Работа посвящена разработке параллельного алгоритма и программного модуля на его основе для решения нестационарных задач теплопроводности (диффузии) в осесимметричной постановке в составе создаваемого авторами программного комплекса. Комплекс предназначен для решения краевых задач для уравнений эллиптического и параболического типа в исследовательских и прикладных целях. Ранее были разработаны параллельные алгоритмы и программы для решения плоских задач теории упругости и теории потенциала методом граничных элементов (МГЭ) $[1,2]$ с применением формул аналитического интегрирования $[3,4]$, которые могут быть использованы для решения, в том числе нелинейных задач [5-10]. Также были разработаны алгоритмы и программы для решения осесимметричных задач теории потенциала и теории упругости $[11,12]$.

В основе разрабатываемых алгоритмов лежит метод граничных элементов, являющийся эффективным инструментом для анализа рассматриваемых задач, и имеющий хороший потенциал распараллеливания вычислений. Подходы с применением МГЭ для решения нестационарных задач теплопроводности впервые были предложены в работах [13-16]. Например, в работе [13] авторы использовали интегральное преобразованием Лапласа в комбинации с методом граничных элементов. После выполнения преобразования Лапласа про- 
изводная по времени исключалась, и исходное уравнение параболического типа для оригинала функции сводилось к более простому уравнению эллиптического типа для изображения функции. Далее граничное интегральное уравнение решалось относительно изображения, и осуществлялся обратный переход к оригиналу искомой функции. Позднее этот подход был использован в работах $[17,18]$. В работе [14] Шоу исследовал трехмерные задачи нестационарной теплопроводности, применяя зависящее от времени фундаментальное решение и рассматривая, в основном, аналитические характеристики метода. Другой подход, основанный на применении метода граничных элементов в сочетании с методом конечных разностей, был предложен Бреббия и Уокером [15]. Вроубел и Бреббия [16] предложили численную методику для решения осесимметричных задач теплопроводности, основанную на представлении фундаментального решения в виде степенного ряда с последующим аналитическим интегрированием по времени интегралов, входящих в граничное интегральное уравнение. Основные идеи решения осесимметричных задач МГЭ были обобщены в книге [19].

В работе рассмотрена вычислительная методика, основанная на использовании зависящего от времени трехмерного фундаментального решения и шаговой по времени схемы численного решения задачи с помощью МГЭ.

\section{2. Математическая постановка задачи}

Рассмотрим уравнение теплопроводности (диффузии) для осесимметричной области $\Omega$

$$
\Delta u(\mathbf{x}, t)-\frac{1}{k} \frac{\partial u(\mathbf{x}, t)}{\partial t}=0, \quad x \in \Omega
$$

Здесь искомая функция $u(\mathbf{x}, t)$ - температура (концентрация) в точке $\mathbf{x}(x, y, z)$ в момент времени $t \in\left[t_{0}, t_{*}\right] ; \Delta$ - оператор Лапласа. Не зависящий от координат и от времени коэффициент $k$ может принимать различные значения в зависимости от рассматриваемой физической задачи.

На поверхности $\Gamma=\Gamma_{1} \cup \Gamma_{2}$, ограничивающей область $\Omega$, заданы обладающие осевой симметрией граничные условия вида:

$$
u(\mathbf{x}, t)=u^{*}(\mathbf{x}, t), \quad \mathbf{x} \in \Gamma_{1} ; \quad q(\mathbf{x}, t)=q^{*}(\mathbf{x}, t), \quad \mathbf{x} \in \Gamma_{2},
$$

где $q(\mathbf{x}, t)=\partial u(\mathbf{x}, t) / \partial \mathbf{n}(\mathbf{x})-$ плотность теплового (диффузионного) потока; $\mathbf{n}(\mathbf{x})$ - внешняя нормаль к поверхности $Г$; звездочкой отмечены известные функции.

Также известно начальное распределение температуры (концентрации) в области $\Omega$ :

$$
u(\mathbf{x}, t)=u_{0}\left(\mathbf{x}, t_{0}\right), \quad \mathbf{x} \in \Omega .
$$

В случае осевой симметрии задачи уравнение (1) примет в цилиндрической системе координат $(r, \varphi, z)$ следующий вид:

$$
\frac{\partial^{2} u(\mathbf{x}, t)}{\partial r^{2}}+\frac{1}{r} \frac{\partial u(\mathbf{x}, t)}{\partial r}+\frac{\partial^{2} u(\mathbf{x}, t)}{\partial z^{2}}-\frac{1}{k} \frac{\partial u(\mathbf{x}, t)}{\partial t}=0
$$

где $\mathbf{x}(r, z) \in \bar{\Omega}, r$ - полярный радиус, ось $z$ направлена вдоль оси симметрии; $\bar{\Omega}$ - область решения осесимметричной задачи, образующая области $\Omega$, лежащая в полуплоскости $r^{+}-z$. 
Краевые условия задачи (2) и (3) в этом случае запишем в виде:

$$
\begin{gathered}
\left.u(\mathbf{x}, t)\right|_{\mathbf{x} \in \bar{\Gamma}_{1}}=u^{*}(\mathbf{x}, t) ;\left.q(\mathbf{x}, t)\right|_{\mathbf{x} \in \bar{\Gamma}_{2}}=q^{*}(\mathbf{x}, t) ; \\
u(\mathbf{x}, t)=u_{0}\left(\mathbf{x}, t_{0}\right), \quad \mathbf{x} \in \bar{\Omega} .
\end{gathered}
$$

Здесь $\bar{\Gamma}=\bar{\Gamma}_{1} \cup \bar{\Gamma}_{2}-$ образующая поверхности $\Gamma$, полученная при пересечении $\Gamma$ с полуплоскостью $r^{+}-z$.

В силу осесимметричности задачи искомая функция $u(\mathbf{x}, t)$, а также граничные и начальная функции $u^{*}(\mathbf{x}, t), q^{*}(\mathbf{x}, t), u_{0}\left(\mathbf{x}, t_{0}\right)$ не зависят от полярного угла $\varphi$.

\section{3. Алгоритм решения}

В соответствии с методом граничных элементов [19] выполним переход от основных уравнений краевой задачи (4) - (6) к граничному интегральному уравнению, которое запишем в цилиндрической системе координат

$$
\begin{gathered}
\frac{1}{2} u\left(\xi, t_{*}\right)=k \int_{t_{0}}^{t_{*}} \int_{\Gamma} q(\mathbf{x}, t) \int_{0}^{2 \pi} u^{*}\left(\xi, \mathbf{x}, t_{*}, t\right) d \varphi(\mathbf{x}) r(\mathbf{x}) d \bar{\Gamma}(\mathbf{x}) d t- \\
-k \int_{t_{0}}^{t_{*}} \int_{\bar{\Gamma}} u(\mathbf{x}, t) \int_{0}^{2 \pi} q^{*}\left(\xi, \mathbf{x}, t_{*}, t\right) d \varphi(\mathbf{x}) r(\mathbf{x}) d \bar{\Gamma}(\mathbf{x}) d t+\int_{\bar{\Omega}} u_{0}\left(\mathbf{x}, t_{0}\right) \int_{0}^{2 \pi} u^{*}\left(\xi, \mathbf{x}, t_{*}, t\right) d \varphi(\mathbf{x}) r(\mathbf{x}) d \bar{\Omega}(x) .
\end{gathered}
$$

Здесь точка $\xi$ принадлежит граничному контуру $\bar{\Gamma} ; u^{*}\left(\xi, \mathbf{x}, t_{*}, t\right)-$ известное трехмерное фундаментальное решение [19]; $q^{*}\left(\boldsymbol{\xi}, \mathbf{x}, t_{*}, t\right)=\partial u^{*}\left(\xi, \mathbf{x}, t_{*}, t\right) / \partial \mathbf{n}(\mathbf{x})$. Зависящее от времени фундаментальное решение в цилиндрической системе координат имеет следующий вид:

$$
u^{*}\left(\xi, \mathbf{x}, t_{*}, t\right)=\frac{1}{(4 \pi k \tau)^{3 / 2}} \exp \left(-\frac{R^{2}(\xi, \mathbf{x})}{4 k \tau}\right)
$$

где $\tau=t_{*}-t ; R(\xi, \mathbf{x})=r^{2}(\xi)+r^{2}(\mathbf{x})-2 r(\xi) r(\mathbf{x}) \cos [\phi(\xi)-\phi(\mathbf{x})]+[z(\xi)-z(\mathbf{x})]^{2}-$ расстояние от фиксированной точки с координатами $(r(\xi), \phi(\xi), z(\xi))$ до переменной точки с координатами $(r(\mathbf{x}), \phi(\mathbf{x}), z(\mathbf{x}))$.

Введем обозначения и выполним интегрирование:

$$
\begin{aligned}
& \bar{u}^{*}\left(\xi, \mathbf{x}, t_{*}, t\right)=\int_{0}^{2 \pi} u^{*}\left(\xi, \mathbf{x}, t_{*}, t\right) d \varphi(x)=\frac{2 \pi}{(4 \pi k \tau)^{3 / 2}} \exp \left(-\frac{s}{4 k \tau}\right) I_{0}\left(\frac{l}{2 k \tau}\right) ; \\
& \bar{q}^{*}\left(\xi, \mathbf{x}, t_{*}, t\right)=\int_{0}^{2 \pi} q^{*}\left(\xi, \mathbf{x}, t_{*}, t\right) d \varphi(x)=\frac{1}{8 \pi^{1 / 2}(k \tau)^{5 / 2}} \exp \left(-\frac{s}{4 k \tau}\right) \times \\
& \times\left\{\left[r(\mathbf{x}) I_{0}\left(\frac{l}{2 k \tau}\right)-r(\xi) I_{1}\left(\frac{l}{2 k \tau}\right)\right] n_{r}(x)-[z(\xi)-z(\mathbf{x})] I_{0}\left(\frac{l}{2 k \tau}\right) n_{z}(\mathbf{x})\right\} .
\end{aligned}
$$


Здесь $s=r^{2}(\xi)+r^{2}(\mathbf{x})+[z(\xi)-z(\mathbf{x})]^{2} ; l=r(\xi) r(\mathbf{x}) ; I_{0}-$ модифицированная функция Бесселя первого рода нулевого порядка; $I_{1}-$ модифицированная функция Бесселя первого рода первого порядка; $n_{r}(\mathbf{x})$ и $n_{z}(\mathbf{x})$ - проекции вектора нормали на оси цилиндрической системы координат.

После подстановки (9) и (10) в (7) получим окончательное граничное интегральное уравнение для случая осевой симметрии краевой задачи:

$$
\begin{gathered}
\frac{1}{2} u\left(\xi, t_{*}\right)=k \int_{t_{0}}^{t_{*}} \int_{\bar{\Gamma}} q(\mathbf{x}, t) \bar{u}^{*}\left(\xi, \mathbf{x}, t_{*}, t\right) r(\mathbf{x}) d \bar{\Gamma}(\mathbf{x}) d t- \\
-k \int_{t_{0}}^{t_{*}} \int_{\bar{\Gamma}} u(\mathbf{x}, t) \bar{q}^{*}\left(\xi, \mathbf{x}, t_{*}, t\right) r(\mathbf{x}) d \bar{\Gamma}(\mathbf{x}) d t+\int_{\bar{\Omega}} u_{0}\left(\mathbf{x}, t_{0}\right) \bar{u}^{*}\left(\xi, \mathbf{x}, t_{*}, t\right) r(x) \mathbf{x} d \bar{\Omega}(\mathbf{x}) .
\end{gathered}
$$

Для получения численного решения граничного интегрального уравнения (11) был использован прием шагового интегрирования по времени в соответствии со следующей схемой: каждый раз процесс интегрирования по времени начинался с момента времени $t_{0}$, решение находили последовательно через определенные шаги $h=t_{f}-t_{f-1}, f=1,2, \ldots, F$, число промежуточных шагов возрастало с течением времени до достижения окончательного момента времени $t_{*}$. Кроме того, на каждом временном шаге выполнялась дискретизация граничного контура на $(N+M)$ прямолинейных граничных элементов $e_{i}(i=1, \ldots, M+N)$, когда граница $\bar{\Gamma}_{1}$ разбита на $N$ граничных элементов, а граница $\bar{\Gamma}_{2}-$ на $M$ элементов. Предполагалось, что функции $u(\mathbf{x}, t)$ и $q(\mathbf{x}, t)$ - кусочно-постоянные, т. е. принимают постоянные значения на каждом граничном элементе на каждом шаге по времени:

$$
u\left(\xi_{i}, t_{f}\right)=u_{i f}=\text { const } ; q\left(\xi_{i}, t_{f}\right)=q_{i f}=\text { const }, i=1, \ldots, 2 N+M, f=1,2, \ldots, F,
$$

где $\xi_{i}$ - узел, находящийся в середине граничного элемента $e_{i}$.

Наличие начальных условий приводит к интегралу по области $\bar{\Omega}$ (последнему слагаемому в правой части уравнения (11)). Для вычисления этого интеграла область разбивали на $L$ конечных элементов: $\Delta \bar{\Omega}_{1}, \Delta \bar{\Omega}_{2}, \ldots, \Delta \bar{\Omega}_{L}$, в каждом из которых функция $u_{0}\left(\mathbf{x}_{l}, t_{0}\right)$ полагалась постоянной, затем выполняли численное интегрирование по каждому из конечных элементов.

$$
\int_{\Omega} u_{0}\left(\mathbf{x}, t_{0}\right) \bar{u}^{*}\left(\xi, \mathbf{x}, t_{F}, t\right) r(\mathbf{x}) d \bar{\Omega}(\mathbf{x})=\sum_{l=1}^{L} u_{0}\left(\mathbf{x}_{l}, t_{0}\right) \int_{\Delta \bar{\Omega}_{l}} \bar{u}^{*}\left(\xi, v, t_{F}, t\right) r(\mathbf{x}) d \bar{\Omega}_{l}(\mathbf{x}) .
$$

Здесь $\mathbf{x}_{l}-$ узел элемента $\Delta \bar{\Omega}_{l}$.

Описанная дискретизация приводит граничное интегральное уравнение (11) к следующему виду:

$$
\frac{1}{2} u\left(\xi_{j}, t_{F}\right)=k \sum_{i=1}^{N} \sum_{f=1}^{F}\left(q_{i f} \int_{e_{i}} \int_{t_{f-1}}^{t_{f}} \bar{u}^{*}\left(\xi_{j}, \mathbf{x}, t_{F}, t\right) r(\mathbf{x}) d t d \bar{\Gamma}(\mathbf{x})-u_{i f}^{*} \int_{e_{i}}^{t_{t_{f-1}}} \int_{\bar{q}}^{*}\left(\xi_{j}, \mathbf{x}, t_{F}, t\right) r(\mathbf{x}) d t d \bar{\Gamma}(\mathbf{x})\right)+
$$




$$
\begin{gathered}
+k \sum_{i=N+1}^{M} \sum_{f=1}^{F}\left(q_{i f}^{*} \int_{e_{i} t_{f-1}}^{t_{f}} \bar{u}^{*}\left(\xi_{j}, \mathbf{x}, t_{F}, t\right) r(\mathbf{x}) d t d \bar{\Gamma}(\mathbf{x})-u_{i f} \int_{e_{i} t_{f-1}}^{t_{f}} \bar{q}^{*}\left(\xi_{j}, \mathbf{x}, t_{F}, t\right) r(\mathbf{x}) d t d \bar{\Gamma}(\mathbf{x})\right)+ \\
+\sum_{l=1}^{L} u_{0}\left(x_{l}, t_{0}\right) \int_{\Delta \bar{\Omega}_{l}} \bar{u}^{*}\left(\xi_{j}, \mathbf{x}, t_{F}, t\right) r(\mathbf{x}) d \bar{\Omega}_{l}(\mathbf{x}) .
\end{gathered}
$$

Соотношения (14), записанные для всех граничных узлов, $j=1, \ldots, N+M$, образуют систему линейных алгебраических уравнений (СЛАУ) относительно узловых значений функций температуры (концентрации) и плотности потока, не заданных граничными условиями. Отметим, что решение системы выполняется для момента времени $t=t_{F}$, а значения $u_{i f}$ и $q_{i f}$ при $f=1, \ldots, F-1$ уже известны из решения на предыдущем временном шаге.

После определения всех граничных значений может быть найдено значение искомой функции температуры (концентрации) краевой задачи (4) - (6) в любой внутренней точке $\zeta$ области $\bar{\Omega}$ :

$$
\begin{gathered}
u\left(\zeta, t_{F}\right)=k \sum_{i=1}^{N} \sum_{f=1}^{F}\left(q_{i f} \int_{e_{i}} \int_{t_{f-1}}^{t_{f}} \bar{u}^{*}\left(\zeta, \mathbf{x}, t_{F}, t\right) r(\mathbf{x}) d t d \bar{\Gamma}(\mathbf{x})-u_{i f}^{*} \int_{e_{i} t_{f-1}}^{t_{f}} \bar{q}^{*}\left(\zeta, \mathbf{x}, t_{F}, t\right) r(\mathbf{x}) d t d \bar{\Gamma}(\mathbf{x})\right)+ \\
+k \sum_{i=N+1}^{M} \sum_{f=1}^{F}\left(q_{i f}^{*} \int_{e_{i}}^{t_{f}} \int_{t_{f-1}}^{t_{f}} \bar{u}^{*}\left(\zeta, \mathbf{x}, t_{F}, t\right) r(\mathbf{x}) d t d \bar{\Gamma}(\mathbf{x})-u_{i f} \int_{e_{i}} \int_{t_{f-1}}^{t_{f}} \bar{q}^{*}\left(\zeta, \mathbf{x}, t_{F}, t\right) r(\mathbf{x}) d t d \bar{\Gamma}(\mathbf{x})\right)+ \\
+\sum_{l=1}^{L} u_{0}\left(\mathbf{x}_{l}, t_{0}\right) \int_{\Delta \bar{\Omega}_{l}} \bar{u}^{*}\left(\zeta, \mathbf{x}, t_{F}, t\right) r(\mathbf{x}) d \bar{\Omega}_{l}(\mathbf{x}) .
\end{gathered}
$$

Для эффективной работы вычислительного алгоритма необходимо использовать специально подобранные схемы вычисления интегралов в системе уравнений (14) и уравнении (15). Интегрирование по времени проводилось аналитически с использованием представлений функций Бесселя в виде степенных рядов [20]:

$$
I_{0}(y)=\sum_{n=0}^{\infty} \frac{1}{n !^{2}}\left(\frac{y}{2}\right)^{2 n} ; I_{1}(y)=\sum_{n=0}^{\infty} \frac{1}{n !^{2}(n+1)}\left(\frac{y}{2}\right)^{2 n+1},
$$

а при больших значениях аргумента - асимптотических представлений функций Бесселя [20]:

$$
I_{0}(y)=\frac{\exp (y)}{\sqrt{2 \pi y}}\left(1+\sum_{n=0}^{\infty} \frac{f_{1}(n)}{n !(8 y)^{n}}\right) ; I_{1}(y)=\frac{\exp (y)}{\sqrt{2 \pi y}}\left(1+\sum_{n=0}^{\infty} \frac{f_{2}(n)}{n !(8 y)^{n}}\right),
$$

где $f_{1}(n)=(2 n-1)^{2}(2 n-3)^{2} \ldots 1 ; f_{2}(n)=(-1)^{n}\left(4-(2 n-1)^{2}\right)\left(4-(2 n-3)^{2}\right) \ldots(4-1)$.

Отметим, что на каждом временном шаге решения задачи в последних слагаемых суммы интегралов по времени из уравнений (14) и (15), когда $f=F$, присутствуют сингулярные интегралы. В этом случае интегрирование проводилось после выполнения перехода в подынтегральном выражении $t_{F} \rightarrow t_{F}+\varepsilon$ для бесконечно малой величины $\varepsilon$, т. е. вычисля- 
ли интегралы $\int_{t_{F-1}}^{t_{F}} \bar{u}^{*}\left(\xi_{j}, \mathbf{x},\left(t_{F}+\varepsilon\right), t\right) r(\mathbf{x}) d t$ и $\int_{t_{F-1}}^{t_{F}} \bar{q}^{*}\left(\xi_{j}, \mathbf{x},\left(t_{F}+\varepsilon\right), t\right) r(\mathbf{x}) d t$, а затем осуществлялся переход к пределу при $\varepsilon \rightarrow 0$.

Для численного вычисления интегралов, входящих в уравнения (14), (15), по пространственным координатам использовалась адаптивная формула численного интегрирования Гаусса-Кронрода по 61 точкам [21]. Случай, когда $\xi_{i}$ лежит на граничном элементе $\varepsilon_{i}$, требует отдельного рассмотрения, так как при $i=j$ в интегралах возникает сингулярность. Для вычисления таких интегралов были подобраны процедуры, использующие формулы адаптивного интегрирования [22].

Разработанный алгоритм позволяет получить на каждом шаге по времени решение задачи (4)-(6) вида (15), непрерывное по пространственным переменным в области $\bar{\Omega}$.

\section{4. Программная реализация}

Представленный вычислительный алгоритм был реализован в виде программного модуля. Программа была написана на языке программирования $\mathrm{C}++$. Возможности программы позволяют оперативно изменять входные данные, а именно: значения физических параметров задачи, шаг по времени, количество граничных элементов, а также производить компиляцию непосредственно перед выполнением расчета. Для выполнения численных расчетов были использованы библиотека GSL [23] и собрание библиотек классов BOOST [24]. Интегралы по элементам, содержащим точки сингулярности, вычисляли с использованием адаптивных алгоритмов численного интегрирования, учитывающих известные координаты особых точек, реализованных в библиотеке GSL. Для повышения эффективности выполнения программного модуля был выбран открытый стандарт распараллеливания OpenMP $[25,26]$ как наиболее оптимальный для проведения трудоемких и независимых вычислений интегралов, входящих в уравнения (14) и (15). Это позволило с использованием многопоточности организовать параллельные вычисления на многопроцессорных вычислительных комплексах с общей оперативной памятью. Известно, что основным недостатком МГЭ является большая потребность в оперативной памяти, вытекающая из высокой плотности матрицы СЛАУ. Однако, в отличие от многих других, этот метод допускает выполнение параллельных вычислений. Процесс построения СЛАУ выполнялся параллельно за счет возможности вычислять коэффициенты матрицы системы полностью независимо друг от друга. Для решения СЛАУ использовалась методика LU-разложения, допускающая распараллеливание. Вычисление значений искомой функции температуры (концентрации) в заданных точках также выполнялось параллельно. Таким образом, программа была распараллелена на каждом этапе решения задачи.

\section{Пример}

Для тестирования алгоритма и его программной реализации были проведены численные исследования. Рассмотрена задача теплопроводности для сплошного кругового цилиндра длиной $2 l$ и диаметром $2 a$, начальная температура которого равна $u_{0}$. В начальный момент времени он помещался в среду с постоянной температурой $u^{*}$. Таким образом, начальные и граничные условия задачи имеют вид:

$$
\begin{gathered}
u_{0}\left(r, z, t_{0}\right)=u_{0} ; \\
\left.u(r, z, t)\right|_{r=a}=u^{*} ;\left.u(r, z, t)\right|_{z= \pm l}=u^{*} .
\end{gathered}
$$


Требуется найти распределение температуры внутри цилиндра в различные моменты времени при условии осевой симметрии задачи. Заметим, что в силу симметрии достаточно рассматривать лишь половину поперечного сечения цилиндра.

Точное решение задачи (4), (18), (19) известно и имеет следующий вид [27]:

$$
u(x, t)=u^{*}+\left(u_{0}-u^{*}\right)\left(\sum_{n=1}^{\infty} \sum_{m=1}^{\infty} A_{n} A_{m} I_{0}\left(\mu_{n} \frac{r}{a}\right) \cos \mu_{m} \frac{z}{l} \cdot \exp \left(-\left(\mu_{n}^{2}+\mu_{m}^{2} K_{l}^{2}\right) \mathbf{F}_{0}\right)\right),
$$

где $A_{n}=\frac{2}{\mu_{n} I_{1}\left(\mu_{n}\right)} ; \mu_{n}-$ корни функции Бесселя первого рода нулевого порядка; $A_{m}=(-1)^{m+1} \frac{2}{\mu_{m}} ; \mu_{m}=(2 m-1) \frac{\pi}{2} ; K_{l}=\frac{a}{l} ; \mathbf{F}_{0}=\frac{k t}{a^{2}}$.

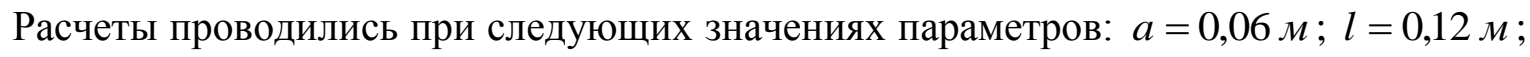
$k=1,25 \cdot 10^{-5} \boldsymbol{M}^{2} / c ; u_{0}=0 ; u^{*}=20 \mathrm{~K}$.

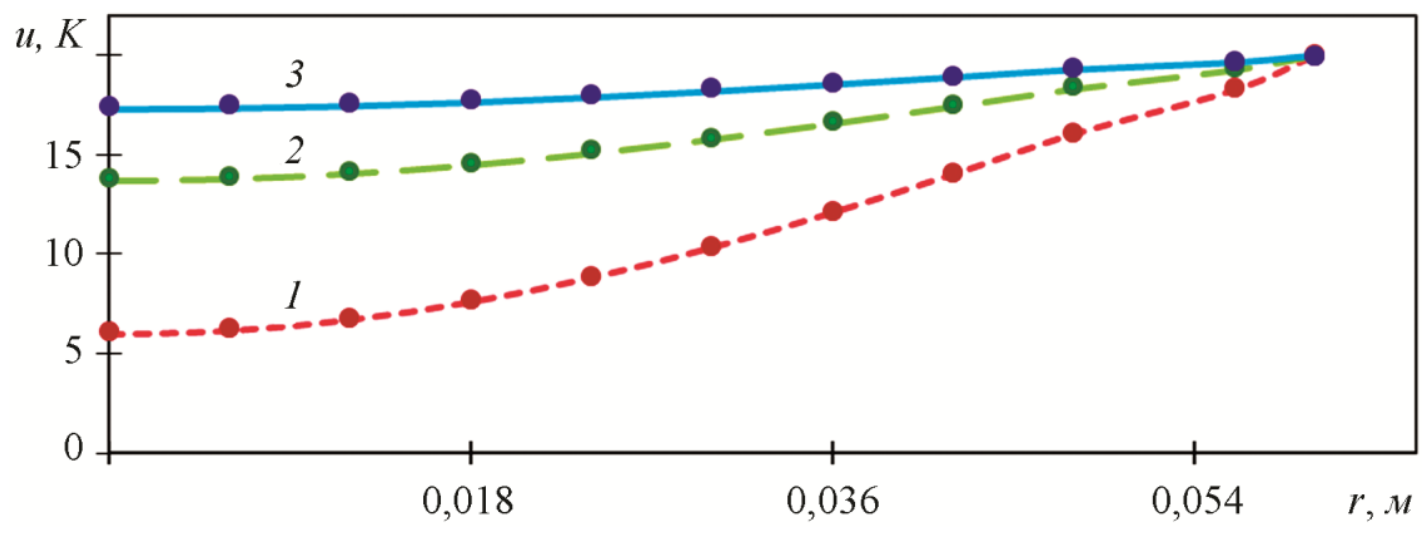

Сравнение численного и точного решений: $1-t=40 c, 2-t=80 c$, $3-t=120 c$; линии - численное, точки - точное

На рисунке приведено сравнение точного решения и решения МГЭ с шагом по времени $h=10 c$ в различные моменты времени: $t_{*}=40 c ; t_{*}=80 c ; t_{*}=120 c$. Значения температуры вдоль радиуса цилиндра, полученные при использовании МГЭ, оказались близки к точному решению (20), что говорит об эффективности предложенной технологии решения.

\section{5. Заключение}

Разработан программный модуль для решения нестационарных задач теплопроводности и диффузии в осесимметричных областях при осесимметричных граничных условиях. В ходе работы была использована методика распараллеливания алгоритма на основе метода граничных элементов для открытого стандарта OpenMP. Проведенное тестирование программы показало достаточную близость результатов расчетов к известному точному решению. Разработанный модуль войдет в программный комплекс для решения задач математической физики, существенно расширив класс решаемых задач.

\section{Благодарность}

Работа выполнена в рамках государственного задания, номер государственной регистраџии АAAA-A18-118020790140-5. 


\section{Литература}

1. Федотов В. П., Спевак Л. Ф., Нефедова О. А. Параллельные алгоритмы для анализа прочности наводороженных конструкций // Программные продукты и системы. - 2012. T. 99, № 3. - С. 235-239.

2. Федотов В. П., Спевак Л. Ф., Нефедова О. А. Программный комплекс для решения задач теории потенциала методом граничных элементов // Программные продукты и системы. - 2014. - Т. 108, № 4. - С. 178-183. - DOI: 10.15827/0236-235X.108.178-182.

3. Федотов В. П., Спевак Л. Ф. Аналитическое интегрирование функций влияния для решения задач упругости и теории потенциала методом граничных элементов // Математическое моделирование. - 2007. - Т. 19, № 2. - С. 87-104.

4. Fedotov V. P., Spevak L. F. One approach to the derivation of exact integration formulae in the boundary element method // Engineering Analysis with Boundary Elements. - 2008. - Vol. 32, No. 10. - P. 883-888. - DOI: 10.1016/j.enganabound.2008.03.001.

5. Федотов В. П., Спевак Л. Ф., Нефедова О. А. Моделирование процессов упругопластического деформирования модифицированным методом граничных элементов // Программные продукты и системы. - 2013. - Т. 4, № 4. - С. 253-257.

6. Spevak L. F., Nefedova O. A. Solving a two-dimensional nonlinear heat conduction equation with degeneration by the boundary element method with the application of the dual reciprocity method // AIP Conference Proceedings. - 2016. - Vol. 1785. - P. 040077. DOI: $10.1063 / 1.4967134$.

7. Spevak L. F., Nefedova O. A. Solving a two-dimensional nonlinear heat conduction equation with nonzero boundary conditions by the boundary element method // AIP Conference Proceedings. - 2017. - Vol. 1915. - P. 040055. - DOI: 10.1063/1.5017403.

8. Kazakov A. L., Nefedova O. A., Spevak L. F. Solution of the Problem of Initiating the Heat Wave for a Nonlinear Heat Conduction Equation Using the Boundary Element Method // Computational Mathematics and Mathematical Physics. - 2019. - Vol. 59, iss. 6. - P. 1015-1029. DOI: 10.1134/S0965542519060083.

9. On the Analytical and Numerical Study of a Two-Dimensional Nonlinear Heat Equation with a Source Term / A. Kazakov, L. Spevak, O. Nefedova, A. Lempert // Symmetry. - 2020. Vol. 12, iss. 6. - article 921. - DOI: 10.3390/sym12060921.

10. Kazakov A. L., Spevak L. F., Nefedova O. A. A Numerical Solution to the TwoDimensional Nonlinear Degenerate Heat Conduction Equation with a Source // AIP Conference Proceedings. - 2020. - Vol. 2315. - P. 040018. - DOI: 10.1063/5.0036718.

11. Spevak L. F., Nefedova O. A. Parallel technology for solving the poisson equation in axisymmetric domains by the boundary element method // AIP Conference Proceedings. - 2018. Vol. 2053. - P. 030070. - DOI: 10.1063/1.5084431.

12. Nefedova O. A., Spevak L. F. Parallel Technology for Solving Axisymmetric Problems of the Theory of Elasticity by the Boundary Element Method // AIP Conference Proceedings. - 2020. Vol. 2315. - P. 020030. - DOI: 10.1063/5.0037021.

13. Rizzo F. J., Shippv, D. J. A method of solution for certain problems of transient heat conduction // IAA J. - 1970. - Vol. 8. - P. 2004-2009. - DOI:10.2514/3.6038.

14. Shaw R. P. An integral equation approach to diffusion // International Journal of Heat and Mass Transfer. - 1974. - Vol. 17. - P. 693-699. - DOI: 10.1016/0017-9310(74)90202-6.

15. Brebbia C. A., Walker S. Boundary Element Techniques in Engineering. - London : Newnes-Butterworths, 1980. - ISBN: 9781483102566.

16. Wrobel L. C., Brebbia C. A. A formulation of the boundary element method for axisymmetric transient heat conduction // International Journal of Heat and Mass Transfer. - 1981. Vol. 24. - P. 843-850. - DOI: 10.1016/S0017-9310(81)80007-5. 
17. Zhu S. P. Time-dependent reaction diffusion problems and the LTDRM approach // Boundary Integral Methods: Numerical and Mathematical Aspects. Southampton / ed. by M. Goldberg. - Boston : Computational Mechanics Publications, 1999. - P. 1-35.

18. Sutradhar A., Paulino G. H, Gray L. J. Transient heat conduction in homogeneous and nonhomogeneous materials by the Laplace Transform Galerkin boundary element method // Engineering Analysis with Boundary Elements. - 2002. - Vol. 26. - P. 119-132. DOI: 10.1016/S0955-7997(01)00090-X.

19. Brebbia C. A., Telles J. F. C., Wrobel L. C. Boundary Element Techniques. - Berlin, Nei-delberg, New-York, Tokyo : Springer-Verlag, 1984. - 466 p. - ISBN 978-3-642-48862-7. DOI: $10.1007 / 978-3-642-48860-3$.

20. Abramowitz M., Stegun I. A. Handbook of Mathematical Functions With Formulas, Graphs, and Mathematical Tables // Dover Books on Advanced Mathematics / ed. by M. Abramowitz, I. A. Stegun. - New York : Dover Publications, 1965.

21. Кронрод А. С. Узлы и веса квадратурных формул. - М. : Наука, 1964. - 143 с.

22. Лифанов И. К. Метод сингулярных интегральных уравнений и численный эксперимент. - М. : Янус, 1995. - 520 с. - ISBN 5-88929-003-7.

23. GSL-GNU Scientific Library. Available at: http://www.gnu.org/software/gsl/ (accessed 07.04.2021).

24. Boost C++ Libraries. Available at: http://www.boost.org/ (accessed 07.04.2021).

25. What is OpenMP? PARALLEL.RU. Available at: https://parallel.ru/tech/tech_dev/openmp.html (accessed 11.05.2021).

26. OpenMP. Available at: http://www.openmp.org/ (accessed 11.05.2021).

27. Лыков А. В. Теория теплопроводности. - М. : Высшая школа, 1967. - 597 с. 